\title{
Robust Current Estimation Applied to the Multiloop Resonant Control of Uninterruptible Power Supplies *
}

\author{
V. C. da Cunha, J. V. Flores, L.F.A. Pereira, A.T. Salton, \\ J. M. Gomes da Silva Jr. \\ School of Engineering, Universidade Federal do Rio Grande do Sul, \\ Brazil (email: \{vicente.cunha, jeferson.flores, lfpereira, aurelio.salton, \\ jmgomes\}@ufrgs.br).
}

\begin{abstract}
This paper addresses the use of inductor current estimators in the multiloop control of uninterruptible power supplies (UPS), assuming that only the output voltage is available for feedback and taking into account the robustness against load variations. Resonant and proportional controllers are assumed to regulate the voltage and inductor current loops, respectively. Conditions to independently design estimator and controller gains are provided, allowing the use of the proposed estimator in preexisting control schemes. Closed-loop stability under estimated current feedback is assessed a posteriori. Simulation results for a single-phase $3.5 \mathrm{kVA}$ UPS show compliance with the IEC 62040 standard.
\end{abstract}

Keywords: Uninterruptible Power Supply, Current Estimation, Robust Control.

\section{INTRODUCTION}

Uninterruptible power supplies (UPS) are power electronic devices with the primary purpose of maintaining the continuity of a power source, safeguarding its load against short-term outages. Depending on design, UPS may also be able to provide regulated power quality by keeping the supplied voltage characteristics (e.g. frequency, amplitude, harmonic distortion) within desirable ranges. UPS performance can be assessed in terms of industrial normative standards, such as the IEC 62040-3 (2011).

In terms of control design, the UPS output voltage should track a sinusoidal reference of specific frequency and amplitude, while rejecting disturbances with high harmonic content. From the theoretical point of view, compensators based on the internal model principle - IMP, are best suited to achieve this goal, such as resonant (Pereira et al., 2014) and repetitive (Escobar et al., 2007) controllers. Most control topologies reported in the literature (Lee et al., 2015; Xu et al., 2013; Ryan et al., 1997) make use of multiloop structures that include the feedback of inductor current to improve the overall transient performance.

The feedback of inductor current implies the need of a current sensor, which may be implemented in a variety of ways. Nevertheless, the most relevant requirement for high-power UPS is that the sensor should be electrically isolated from the measured conductor. Most off-the-shelf sensors are based on the Hall effect, usually mounted around the measured conductor. However, one may point

\footnotetext{
* This study was financed in part by the Coordenação de Aperfeiçoamento de Pessoal de Nível Superior - Brasil (CAPES) - Finance Code 001 and CNPq (Brazil), grant numbers: PQ-306223/20180, PQ-302675/2018-3, PQ-306214/2018-0, PQ-305979/2015-9, Univ422992/2016-0.
}

out a number of issues inherent to these sensors, e.g., its positioning dependence, temperature drift and measurement errors due to magnetization (Ziegler et al., 2009). Also, the use of analog to digital converters for data acquisition and control introduces quantization noise. Finally, UPS control is implemented through semiconductor switching, which may also induce high frequency noise (Sangswang and Nwankpa, 2004).

Besides having to account for the mentioned noise issues, another major design concern for UPS control is related to load uncertainty. From a designer perspective, the only available specification for load is its maximum power consumption, which translates into a range of possible load admittance values. The standard Kalman filter approach for systems with an uncertain parameter consists in assuming a stochastic process model for the uncertain parameter and taking it as an additional state to be estimated (Jazwinski, 1970). Another possible estimation strategy is similar to "high-gain" filtering (Prasov and Khalil, 2013): specify a nominal model for the system and assume the load variation as additional process noise, therefore increasing the Kalman filter gain and the estimation responsiveness to measurements. To keep computational requirements low, a non-adaptive formulation of the Kalman filter can be used at the expense of estimation performance degradation (Simon, 2006), using only the steady-state filter gain instead of periodically recalculating it. Besides that, one could consider an estimator design procedure that is robust to load uncertainty, so from these arguments, (Petersen and McFarlane, 1994; Xie and Soh, 1994) present a steady-state Kalman filter extension to systems with norm-bounded uncertainties. Both Petersen and McFarlane (1994) and Xie and Soh (1994), using slightly different approaches, obtained an estimator with an upper-bound on the estimation error covariance by the solution of two 
continuous-time algebraic Ricatti equations (CAREs) that are coupled by a scaling parameter.

This paper proposes a robust design procedure for the multiloop resonant control of a single-phase UPS assuming that only the UPS output voltage is measured. The proposed controller structure is based on a resonant controller to regulate the voltage loop and a proportional inductor current feedback to improve transient performance. Assuming a norm-bounded modeling of the uncertain load connected to the UPS output, the robust steady-state Kalman filter presented in Petersen and McFarlane (1994) is employed for inductor current estimation. A three-step design procedure is considered: i) controller design is carried out in terms of an optimization problem under linear matrix inequality (LMI) constraints that guarantee closedloop performance in transient and steady-state; ii) an inductor current estimator is computed by the solution of an optimization problem with CARE constraints that minimize an upper bound for the estimation error covariance; iii) closed-loop stability under the estimated inductor current is verified a posteriori. The proposed method is then validated in PSIM for a $3.5 \mathrm{kVa}$ single phase commercial UPS in terms of the IEC 62040 performance requirements.

Notation: $\operatorname{tr}\{X\}$ denotes the trace of matrix $X$ and $\mathrm{E}\{x\}$ the expected value of $x ; \operatorname{diag}\{X, Y\}$ is a block diagonal matrix with $\mathrm{X}$ and $\mathrm{Y}$ in the main diagonal; $\operatorname{He}\{X\} \triangleq X+$ $X^{\mathrm{T}} \cdot \mathcal{R}\{s\}$ denotes the real part of $s$.

\section{AVERAGE MODEL OF UPS OUTPUT STAGE}

In most applications, online double-conversion UPS are composed of an input rectifier, a DC energy storage device and an output inverter. Only the output inverter will be considered in this paper, while the rectifier and energy storage stages are assumed to provide a DC voltage referred to as "DC link". Fig. 1 illustrates the output stage as comprising a half-bridge inverter and a LC low-pass filter (Pereira et al., 2014).

A common approach for obtaining a linear model of switched converters is to use an averaged state-space model for the circuit over a switching period, also known as state-space averaging Middlebrook and Cuk (1976). From Fig. 1 and assuming a PWM high frequency switching, then the control input $u(t)$ can be defined as the averaged input voltage of the low-pass filter, which is proportional to the switching duty-cycle. With the connection of a time-varying load with admittance $Y_{\text {load }}(t)$ and a current source $i_{d}(t)$, the equivalent circuit for the averaged model is presented in Fig. 2. Disturbances in the DC link (such as ripple voltage) are modeled by $v_{d}(t)$. The output current

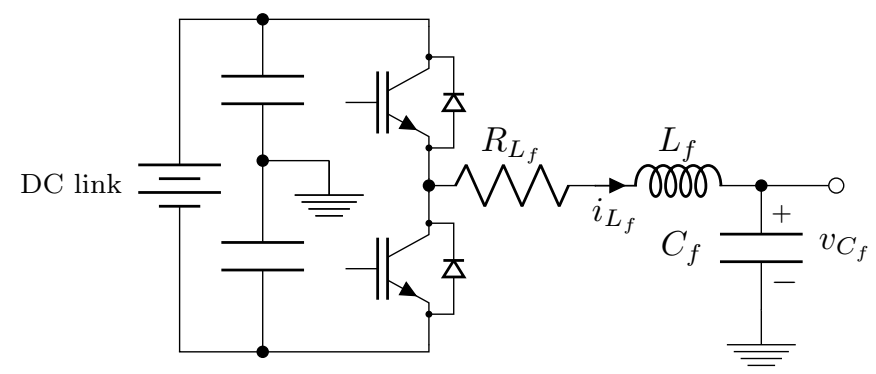

Figure 1. The output stage of UPS.

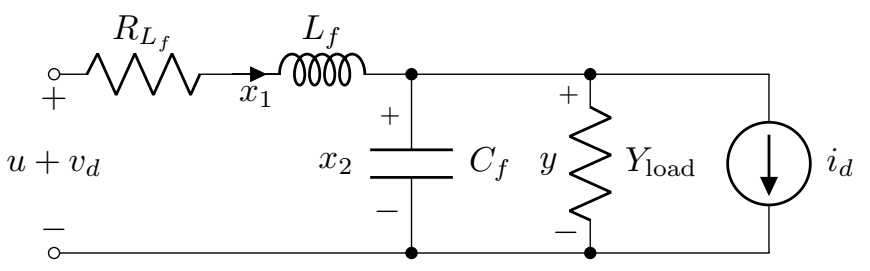

Figure 2. Average model of UPS output stage with load.

disturbance $i_{d}(t)$ mainly represents the harmonic content of nonlinear loads, but both $v_{d}(t)$ and $i_{d}(t)$ may include other unmodeled disturbances as well (Pereira et al., 2014).

As UPS are not designed for specific loads, but rather for any load up to a maximum power, the load admittance is uncertain but restricted to the interval $Y_{\min } \leq Y_{\text {load }}(t) \leq$ $Y_{\max }$. This range can be rewritten in terms of a timevarying parameter $\delta(t)$, as follows:

$$
\begin{gathered}
Y_{\text {load }}(t)=Y_{0}+\delta(t) \frac{Y_{\min }-Y_{\max }}{2} \\
Y_{0}:=\frac{Y_{\max }+Y_{\min }}{2}, \quad\|\delta(t)\| \leq 1
\end{gathered}
$$

The state-space representation of the UPS output stage average model is then given by

$$
\begin{aligned}
& \left\{\begin{array}{l}
\dot{x}(t)=\left[A+B_{\delta} \delta(t) C_{\delta}\right] x(t)+B u(t)+B_{w} w(t) \\
y(t)=C x(t) \quad e(t)=r(t)-y(t)
\end{array}\right. \\
& A=\left[\begin{array}{cc}
-R_{L_{f}} / L_{f} & -1 / L_{f} \\
1 / C_{f} & -Y_{0} / C_{f}
\end{array}\right] \quad B_{\delta}=\left[\begin{array}{c}
0 \\
\frac{Y_{\max }-Y_{\min }}{2 C_{f}}
\end{array}\right] \\
& C_{\delta}=\left[\begin{array}{ll}
0 & 1
\end{array}\right] \quad B=\left[\begin{array}{c}
1 / L_{f} \\
0
\end{array}\right] \quad B_{w}=\left[\begin{array}{cc}
1 / L_{f} & 0 \\
0 & -1 / C_{f}
\end{array}\right] \\
& C=\left[\begin{array}{ll}
0 & 1
\end{array}\right] \quad w(t):=\left[v_{d}(t) i_{d}(t)\right]^{\mathrm{T}}
\end{aligned}
$$

where $x(t):=\left[x_{1}(t) x_{2}(t)\right]^{\mathrm{T}}$, with $x_{1}(t)$ and $x_{2}(t)$ being, respectively, the averaged inductor current and capacitor voltage.

\section{MULTILOOP RESONANT CONTROL DESIGN}

In the sequel, a design method for the state-feedback multiloop resonant controller is proposed, corresponding to step i) mentioned in section 1.

Consider a transfer function

$$
G_{\omega_{0}}(s)=\frac{\left(\alpha_{\omega_{0}}+\beta_{\omega_{0}}\right) s+\left(\alpha_{\omega_{0}}-\beta_{\omega_{0}}\right) \omega_{0}}{s^{2}+\omega_{0}^{2}}+\gamma_{\omega_{0}}
$$

where $\omega_{0}$ is a given resonance frequency and $\alpha_{\omega_{0}}, \beta_{\omega_{0}}$, $\gamma_{\omega_{0}}$ are constants. From the IMP, the inclusion of $G_{\omega_{0}}(s)$ in the direct path between tracking error $e(t)$ and plant input $u(t)$ guarantees asymptotic reference tracking for a sinusoidal reference signal $r(t)$ with frequency $\omega_{0}$, as long as $\alpha_{\omega_{0}}, \beta_{\omega_{0}}, \gamma_{\omega_{0}}$ are tuned to ensure the closedloop stability. In control literature this structure is usually referred as a resonant controller (Pereira et al., 2014).

The IMP also guarantees asymptotic disturbance rejection if plant disturbances $w(t)$ are sinusoids with the same frequency as $r(t)$. However, this is not the case when dealing with nonlinear loads connected to the UPS output, since the output current disturbance $i_{d}(t)$ will contain harmonic components which are odd-multiples of $\omega_{0}$. To 
achieve the disturbance rejection of $N$ odd harmonics, (5) can be expanded to include $N$ resonance modes, as follows:

$$
G_{c}(s)=G_{\omega_{0}}(s)+G_{3 \omega_{0}}(s)+\cdots+G_{(2 N-1) \omega_{0}}(s),
$$

which can be written in state-space by:

$$
\begin{aligned}
&\left\{\begin{array}{l}
\dot{x}_{c}(t)=A_{c} x_{c}(t)+B_{c} e(t) \\
y_{c}(t)=C_{c} x(t)+D_{c} e(t)
\end{array}\right. \\
& A_{c}= \operatorname{diag}\left\{A_{\omega_{0}}, A_{3 \omega_{0}}, \ldots, A_{(2 N-1) \omega_{0}}\right\} \\
& B_{c}= {\left[\begin{array}{lll}
B_{\omega_{0}}^{\mathrm{T}} & B_{3 \omega_{0}}^{\mathrm{T}} \ldots B_{(2 N-1) \omega_{0}}^{\mathrm{T}}
\end{array}\right]^{\mathrm{T}} } \\
& C_{c}=\left[\begin{array}{lll}
C_{\omega_{0}} & C_{3 \omega_{0}} \ldots C_{(2 N-1) \omega_{0}}
\end{array}\right] \\
& D_{c}=\gamma_{\omega_{0}}+\gamma_{3 \omega_{0}}+\cdots+\gamma_{(2 N-1) \omega_{0}} \\
& A_{n \omega_{0}}:=\left[\begin{array}{cc}
0 & n \omega_{0} \\
-n \omega_{0} & 0
\end{array}\right] \quad B_{n \omega_{0}}:=\left[\begin{array}{l}
1 \\
1
\end{array}\right] \\
& C_{n \omega_{0}}:=\left[\begin{array}{ll}
\alpha_{n \omega_{0}} & \beta_{n \omega_{0}}
\end{array}\right] .
\end{aligned}
$$

Assuming now a multiloop control structure with (7) regulating the voltage loop and a proportional controller with gain $K_{i}$ in the current loop, then the control signal $u(t)$ is defined as

$$
u(t):=y_{c}(t)+K_{i} x_{1}(t) .
$$

This particular control structure results in the closed-loop block diagram of Fig. 3, where the block named UPS refers to the output stage depicted in Fig. 2.

The closed-loop interconnection between the plant and controller can be represented as follows:

$$
\begin{aligned}
& \left\{\begin{array}{r}
\dot{x}_{a}(t)=\left[A_{a}+B_{\Delta} \delta(t) C_{\Delta}\right] x_{a}(t)+B_{a} u(t)+ \\
y(t)=C_{a} x_{a}(t) \\
+B_{W} w(t)+B_{r} r(t)
\end{array}\right. \\
& x_{a}(t):=\left[\begin{array}{c}
x(t) \\
x_{c}(t)
\end{array}\right] \quad A_{a}=\left[\begin{array}{cc}
A & 0 \\
-B_{c} C & A_{c}
\end{array}\right] \\
& B_{\Delta}=\left[\begin{array}{c}
B_{\delta} \\
0
\end{array}\right] \quad C_{\Delta}=\left[\begin{array}{ll}
C_{\delta} & 0
\end{array}\right] \quad B_{a}=\left[\begin{array}{c}
B \\
0
\end{array}\right] \\
& B_{W}=\left[\begin{array}{c}
B_{w} \\
0
\end{array}\right] \quad B_{r}=\left[\begin{array}{c}
0 \\
B_{c}
\end{array}\right] \quad C_{a}=\left[\begin{array}{ll}
C & 0
\end{array}\right]
\end{aligned}
$$

with

$$
u(t)=K x_{a}(t)+D_{c} r(t)
$$

and $K=\left[K_{i}-D_{c} C_{c}\right]$.

To address the performance constraints imposed by UPS standards, the design of gain $K$ must consider the following control objectives:

- the closed-loop system should be robustly $\mathcal{D}$-stable (i.e. eigenvalues of $A_{a}+B_{\Delta} \delta(t) C_{\Delta}+B_{a} K$ should lie in a $\mathcal{D}$-region of the complex plane for all $\|\delta(t)\| \leq 1)$;

- the RMS gain from disturbance $w(t)$ to the regulated output $y(t)$ should be minimized.

The $\mathcal{D}$-region under consideration is the same one as in Pereira et al. (2014), resulting from the intersection of

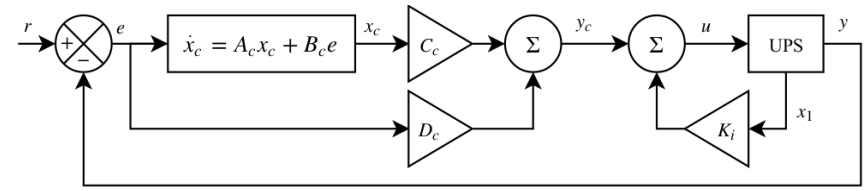

Figure 3. UPS control loop using sensor measurements. a left half-plane $(\mathcal{R}\{s\} \leq-\sigma)$ with an origin-centered disk $(\|s\| \leq \rho)$. The choice of $\mathcal{D}$-region with real part boundary $-\sigma$ imposes a minimum exponential decay rate to the closed-loop state trajectories, which can be tuned to achieve the desirable transient performance. The disk boundary $\rho$ limits the maximum eigenvalues of $A_{a}+$ $B_{\Delta} \delta(t) C_{\Delta}+B_{a} K$, which helps to avoid an arbitrarily high control signal.

LMI constraints for the quadratic $\mathcal{D}$-stability of the system are given in the following theorem:

Theorem 1. If there exists $R=R^{\mathrm{T}}>0, Y=K R, \mu_{1} \geq 0$, $\mu_{2} \geq 0$ that satisfy the following LMI constraints:

$$
\begin{gathered}
{\left[\begin{array}{cc}
X_{2} & R C_{\Delta}^{\mathrm{T}} \\
C_{\Delta} R & -\mu_{1}
\end{array}\right]<0} \\
X_{2}:=\operatorname{He}\left\{A_{a} R+B_{a} Y\right\}+2 \sigma R+\mu_{1} B_{\Delta} B_{\Delta}^{\mathrm{T}} \\
{\left[\begin{array}{ccc}
-\rho R+\mu_{2} B_{\Delta} B_{\Delta}^{\mathrm{T}} & A_{a} R+B_{a} Y & 0 \\
R A_{a}^{\mathrm{T}}+Y^{\mathrm{T}} B_{a}^{\mathrm{T}} & -\rho R & R C_{\Delta}^{\mathrm{T}} \\
0 & C_{\Delta} R & -\mu_{2}
\end{array}\right]<0}
\end{gathered}
$$

then system $(11)$ is quadratically $\mathcal{D}$-stable (in the intersection of a half plane to the left of $-\sigma$ and an origin-centered disk with radius $\rho$ ) for all $\|\delta(t)\| \leq 1$.

Proof. The proof of this theorem follows the same procedure presented in Chilali et al. (1999) and will be omitted due to space constraints.

From Boyd et al. (1994), if there exists $R=R^{\mathrm{T}}>0$, $Y=K R, \mu_{3} \geq 0$ and $\gamma \geq 0$ that satisfy the following LMI constraint:

$$
\begin{gathered}
{\left[\begin{array}{ccc}
X_{3} & R C_{\Delta}^{\mathrm{T}} & R C_{a}^{\mathrm{T}} \\
C_{\Delta} R & -\mu_{3} & 0 \\
C_{a} R & 0 & -\gamma^{2}
\end{array}\right] \leq 0} \\
X_{3}:=\operatorname{He}\left\{A_{a} R+B_{a} Y\right\}+B_{W} B_{W}^{\mathrm{T}}+\mu_{3} B_{\Delta} B_{\Delta}^{\mathrm{T}}
\end{gathered}
$$

then the RMS gain of system (11) from $w(t)$ to $y(t)$ will be less than $\gamma$ for all $\|\delta(t)\| \leq 1$. The design of feedback gains $K=Y R^{-1}$ that result in an optimal RMS gain bound $\gamma$ while accounting for quadratic $\mathcal{D}$-stability can then be obtained by solving the optimization problem:

$$
\min _{R, Y, \mu_{1}, \mu_{2}, \mu_{3}} \gamma \text { subject to: }\left\{\begin{array}{l}
(14) \text { to }(18) \\
R=R^{\mathrm{T}}>0 \\
\mu_{1}, \mu_{2}, \mu_{3}, \gamma \geq 0
\end{array}\right.
$$

\section{STATE ESTIMATOR DESIGN}

Based on the assumption that the UPS output voltage is the only state measurement available, the idea is to design a robust state estimator to implement the inductor current feedback loop. With this aim, consider now that the UPS average model (3) presents both measurement and process noise given by $\psi(t)$ and $\phi(t)$, respectively. Hence, the openloop plant is now represented by

$$
\left\{\begin{array}{l}
\dot{x}(t)=\left[A+B_{\delta} \delta(t) C_{\delta}\right] x(t)+B u(t)+\phi(t) \\
y(t)=C x(t)+\psi(t)
\end{array}\right.
$$

where $\phi(t)$ encompasses both the disturbance $w(t)$ and other effects such as modeling errors due to the state-space averaging technique.

In addition, consider a state estimator in the form of

$$
\left\{\begin{array}{l}
\dot{\hat{x}}(t)=A_{e} \hat{x}(t)+B_{e} y(t)+B u(t) \\
\hat{x}_{1}(t)=C_{e} \hat{x}(t) \quad C_{e}=\left[\begin{array}{ll}
1 & 0
\end{array}\right]
\end{array}\right.
$$


where the state estimation error is given by $e_{e}(t):=x(t)-$ $\hat{x}(t)$. Then, the goal is to determine estimator matrices $A_{e}$ and $B_{e}$ such that an upper bound for the estimation error covariance $\mathrm{E}\left\{e_{e}(t)^{\mathrm{T}} e_{e}(t)\right\}$ is minimized.

For the purposes of using the Kalman filter results, the following assumption holds.

Assumption 2. $\phi(t)$ and $\psi(t)$ are mutually uncorrelated zero-mean white gaussian noise processes. $\phi(t)$ has covariance matrix $W$ and $\psi(t)$ has covariance matrix $\mathrm{V}$.

The work of Petersen and McFarlane (1994) extended the steady-state Kalman filter results to systems with norm-bounded uncertainties, providing an upper-bound for the estimation error covariance. The main result of this reference is given under Lemma 3.

Lemma 3. Suppose that the uncertain system (20) is quadratically stable. Then there exists a constant $\bar{\epsilon}>0$ such that for all $\epsilon \in(0, \bar{\epsilon})$ the Riccati equation

$$
A S+S A^{\mathrm{T}}+\epsilon S C_{\delta}^{\mathrm{T}} C_{\delta} S+\frac{1}{\epsilon} B_{\delta} B_{\delta}^{\mathrm{T}}+W=0
$$

has a solution $S=S^{\mathrm{T}}>0$. For any $0<\epsilon \leq \bar{\epsilon}$, the Riccati equation

$$
\begin{aligned}
A Q+Q A^{\mathrm{T}}+\epsilon Q C_{\delta}^{\mathrm{T}} C_{\delta} Q & -Q C^{\mathrm{T}} V^{-1} C Q+ \\
& +\frac{1}{\epsilon} B_{\delta} B_{\delta}^{\mathrm{T}}+W=0
\end{aligned}
$$

also has a solution $Q=Q^{\mathrm{T}}>0$ and $Q \leq S$. Then, a state estimator (21) with matrices

$$
\begin{gathered}
A_{e}=A+\epsilon Q C_{\delta}^{\mathrm{T}} C_{\delta}-B_{e} C \\
B_{e}=Q C^{\mathrm{T}} V^{-1}
\end{gathered}
$$

guarantees that $\mathrm{E}\left\{e_{e}(t)^{\mathrm{T}} e_{e}(t)\right\} \leq \operatorname{tr}\{Q\}$, i.e. $\operatorname{tr}\{Q\}$ is an upper bound of estimation error covariance. In addition, $\operatorname{tr}\{Q\}$ is a convex function of $\epsilon$ over the range $(0, \bar{\epsilon})$.

It can be shown from (4) that all the eigenvalues of $\left[A+B_{\delta} \delta(t) C_{\delta}\right]$ have strictly negative real parts for any $Y_{\min }>0$, i.e., the assumption of quadratic stability of $(20)$ holds true. Firstly, in order to determine a suitable $\bar{\epsilon}$, we consider the following theorem.

Theorem 4. If $\lambda^{*}$ is the solution to the following optimization problem

$$
\begin{gathered}
\lambda^{*}=\min _{\tilde{S}} \lambda \text { subject to: }\left\{\begin{array}{l}
(27) \\
\tilde{S}=\tilde{S}^{\mathrm{T}}>0 \\
\lambda>0
\end{array}\right. \\
{\left[\begin{array}{cr}
\left.A \tilde{S}+\tilde{S} A^{\mathrm{T}}+\lambda B_{\delta} B_{\delta}^{\mathrm{T}}+W \begin{array}{r}
\tilde{S} C_{\delta}^{\mathrm{T}} \\
C_{\delta} \tilde{S}
\end{array}\right]<0
\end{array}\right.}
\end{gathered}
$$

then $\bar{\epsilon}=1 / \lambda^{*}$ is such that, for all $\epsilon \in(0, \bar{\epsilon})$, the Riccati equation (22) has a solution $S=S^{\mathrm{T}}>0$.

Proof. From Petersen et al. (1991), if (22) is to have a solution $S$, then inequality

$$
A \tilde{S}+\tilde{S} A^{\mathrm{T}}+\epsilon \tilde{S} C_{\delta}^{\mathrm{T}} C_{\delta} \tilde{S}+\frac{1}{\epsilon} B_{\delta} B_{\delta}^{\mathrm{T}}+W<0
$$

must also hold for $\tilde{S}>0$. With the change of variable $\lambda=\epsilon^{-1}$ and through the Schur complement, it follows that the satisfaction of LMI (27) implies (28). Since $\lambda$ and $\epsilon$ are inversely proportional, a maximum value for $\epsilon$ that satisfies these constraints can be obtained by finding a minimum for $\lambda$.
After finding $\bar{\epsilon}$, the state estimator (21) can then be designed by solving the optimization problem

$$
\min _{\epsilon, Q} \operatorname{tr}\{Q\} \text { subject to: }\left\{\begin{array}{l}
(23) \\
Q=Q^{\mathrm{T}}>0 \\
0<\epsilon \leq \bar{\epsilon}
\end{array}\right.
$$

\section{CLOSED-LOOP QUADRATIC STABILITY}

The block diagram in Fig. 4 represents the closed-loop system when the inductor current estimate from (21) is considered in the current loop. The state-space representation for this closed-loop system is then given by:

$$
\begin{aligned}
& \left\{\begin{aligned}
& \dot{\bar{x}}(t)=\left[\bar{A}+\bar{B}_{\Delta} \delta(t) \bar{C}_{\Delta}\right] \bar{x}(t)+\bar{B} u(t)+ \\
& y(t)=\bar{C} \bar{x}(t) \\
&+\bar{B}_{W} w(t)+\bar{B}_{r} r(t)
\end{aligned}\right. \\
& \bar{x}(t):=\left[\begin{array}{c}
x(t) \\
x_{c}(t) \\
e_{e}(t)
\end{array}\right] \quad \bar{A}=\left[\begin{array}{ccc}
A & 0 & 0 \\
-B_{c} C & A_{c} & 0 \\
A-A_{e}-B_{e} C & 0 & A_{e}
\end{array}\right] \\
& \bar{B}_{\Delta}=\left[\begin{array}{c}
B_{\delta} \\
0 \\
B_{\delta}
\end{array}\right] \quad \bar{C}_{\Delta}=\left[\begin{array}{lll}
C_{\delta} & 0 & 0
\end{array}\right] \quad \bar{B}=\left[\begin{array}{c}
B \\
0 \\
0
\end{array}\right] \\
& \bar{B}_{W}=\left[\begin{array}{c}
B_{w} \\
0 \\
B_{w}
\end{array}\right] \quad \bar{B}_{r}=\left[\begin{array}{c}
0 \\
B_{c} \\
0
\end{array}\right] \quad \bar{C}=\left[\begin{array}{lll}
C & 0 & 0
\end{array}\right]
\end{aligned}
$$

with control signal $u(t)$ :

$$
\begin{array}{r}
u(t)=\left[\left(K_{i} C_{e}-D_{c} C\right) C_{c}-K_{i} C_{e}\right] \bar{x}(t)+ \\
+D_{c} r(t)=\bar{K} \bar{x}(t)+D_{c} r(t)
\end{array}
$$

In order to assess the internal stability of the final design, the exogenous signals $w(t)$ and $r(t)$ are disregarded since the internal stability of (30) also implies its BIBO stability (Dorf and Bishop, 2000), resulting in

$$
\dot{\bar{x}}(t)=\left[\bar{A}+\bar{B}_{\Delta} \delta(t) \bar{C}_{\Delta}+\bar{B} \bar{K}\right] \bar{x}(t) .
$$

Closed-loop quadratic stability can then be verified if a solution $U=U^{\mathrm{T}}>0$ and $\mu \geq 0$ exists to the feasibility problem with the following LMI constraint (Boyd et al., 1994):

$$
\left[\begin{array}{r}
\operatorname{He}\{\bar{A} U+\bar{B} \bar{K} U\}+\mu \bar{B}_{\Delta} \bar{B}_{\Delta}^{\mathrm{T}} U \bar{C}_{\Delta}^{\mathrm{T}} \\
\bar{C}_{\Delta} U
\end{array}\right]<0 .
$$

Summarizing the method proposed in this paper, we present a step-by-step design procedure for the multiloop resonant control of UPS with inductor current estimation:

i) Solve the optimization problem (19) to obtain the feedback gains $C_{c}, D_{c}$ and $K_{i}$. In this step, the design

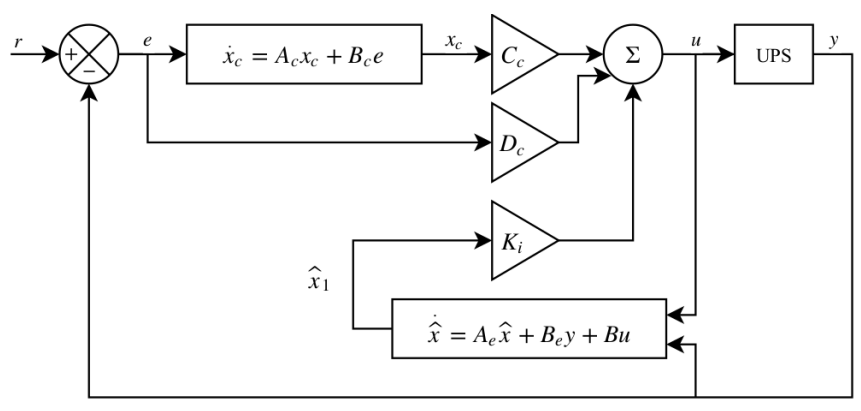

Figure 4. UPS control loop using state estimation. 
parameters are the number of resonance modes $(N)$ and the $\mathcal{D}$-region parameters $(\sigma$ and $\rho$ );

ii) Solve the optimization problem $(26)$ to obtain $\bar{\epsilon}$. With the value of $\bar{\epsilon}$, solve optimization problem (29) and construct estimator matrices $A_{e}$ and $B_{e}$ as in (24) and (25), repectively;

iii) Verify the resulting closed-loop stability by solving the feasibility problem with constraint (34). If stability is not verified, then repeat the previous steps relaxing the $\mathcal{D}$-region or reducing the noise covariance matrices $W$ and $V$.

\section{SIMULATION RESULTS}

In order to illustrate the proposed methodology, consider the $3.5 \mathrm{kVA}$ commercial UPS presented in Corleta et al. (2016) with parameters described in Table 1 and its corresponding nonlinear test loads under the specifications of IEC 62040-3 Commission (2011). With a resonant controller of $N=3$ and a $\mathcal{D}$-stability region with $\sigma=50$ and $\rho=5000$, optimization problem (19) results in $\gamma=$ 1.59 and the following feedback gains:

$$
\begin{aligned}
& K_{i}=-5.19, \quad D_{c}=3.90, \\
& C_{c}=\left[\begin{array}{lllll}
320.10 & 395.80 & 237.41 & 457.47 & 170.59
\end{array}\right.
\end{aligned}
$$

The state estimator is then designed considering the measurement noise covariance matrix $V=0.01$ and process noise covariance matrix $W=1000 I$. The optimization problem (26) yields $\bar{\epsilon}=1.845$ and the solution of $(29)$ results in $\operatorname{tr}\{Q\}=22.83$. The resulting closed-loop system is quadratically stable since (34) is feasible.

To validate the designed controller and estimator, we use a software environment comprising Matlab and PSIM. The controller, state estimator and feedback loop are simulated in Matlab Simulink, while the output stage of Fig. 1 (including the switching inverter) and the test loads are simulated in PSIM. Two simulation setups are considered. The first setup, as depicted in Fig. 3, does not use state estimation, but rather uses a feedback loop with an ideal current sensor. The second setup follows Fig. 4 and employs state estimation. Both setups consider an

Table 1. Parameter values used in simulation

\begin{tabular}{llr} 
Parameter & Symbol & Value \\
\hline Filter inductance & $L_{f}$ & $1 \mathrm{mH}$ \\
Filter inductor resistance & $R_{L_{f}}$ & $15 \mathrm{~m} \Omega$ \\
Filter capacitor & $C_{f}$ & $300 \mu \mathrm{F}$ \\
Minimum load admittance & $Y_{\min }$ & $0.1 \mathrm{mS}$ \\
Maximum load admittance & $Y_{\max }$ & $151.9 \mathrm{mS}$ \\
Reference signal frequency & $\omega_{0}$ & $2 \pi \times 60 \mathrm{rad} / \mathrm{s}$ \\
Reference signal amplitude & - & $127 \sqrt{2} \mathrm{~V}$ \\
DC Link Voltage & - & $520 \mathrm{~V}$ \\
PWM switching frequency & - & $21.6 \mathrm{kHz}$ \\
Nonlinear load series resistor $(25 \%)$ & - & $0.73 \Omega$ \\
Nonlinear load series resistor $(75 \%)$ & - & $0.25 \Omega$ \\
Nonlinear load parallel resistor $(25 \%)$ & - & $41.57 \Omega$ \\
Nonlinear load parallel resistor $(75 \%)$ & - & $13.86 \Omega$ \\
Nonlinear load capacitor $(25 \%)$ & - & $3007 \mu \mathrm{F}$ \\
Nonlinear load capacitor $(75 \%)$ & - & $9021 \mu \mathrm{F}$
\end{tabular}

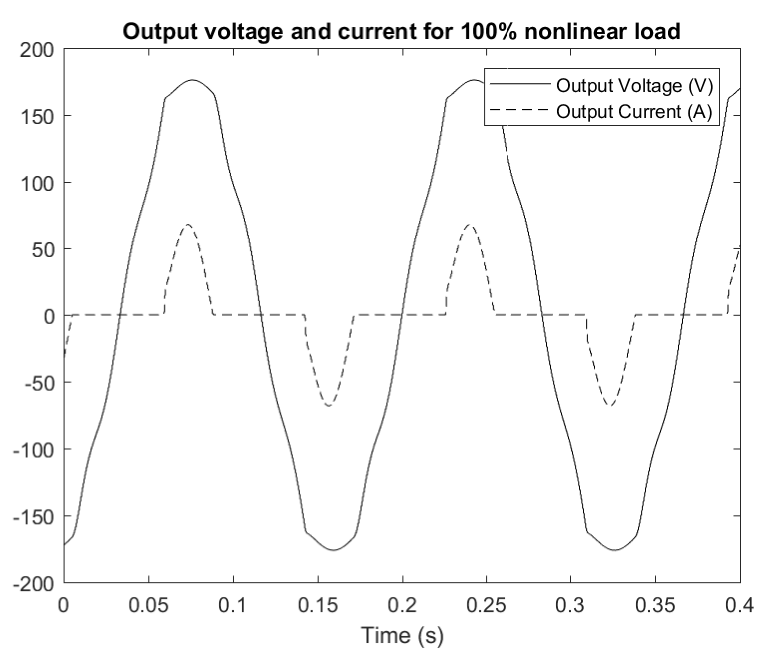

Figure 5. UPS output voltage and current for $100 \%$ nonlinear test load employing the estimated current feedbcak.

output voltage sensor with zero-mean measurement noise of covariance $V=0.01$.

From these setups, Table 2 presents a comparison of the output voltage total/individual harmonic distortion (THD/IHD). As it can be seen, the THD increases when the state estimator is used instead of an ideal sensor, nevertheless remaining within the margins imposed by IEC 62040 . Fig. 5 shows a plot of output voltage and current for the $100 \%$ nonlinear test load using the simulation setup with state estimation.

The simulation of transient performance for nonlinear load steps from $25 \%$ to $100 \%$ nominal value is plotted in Fig. 6 , showing compliance to the most restrictive overvoltage and undervoltage profile presented in the IEC62040. Fig. 7 presents a plot of the estimated inductor current for the different levels of nonlinear load. It can be seen that the overall behavior of the inductor current is correctly estimated by the proposed method.

\section{CONCLUSIONS}

In this work we have presented a systematic design procedure for inductor current estimation in the multiloop resonant control of single-phase UPS. A method based on three independent steps was proposed, where controller and estimator design was carried out by the solution of optimization problems under LMI/CARE constraints and

Table 2. Harmonic distortions of output (100\% nonlinear load)

\begin{tabular}{l|r|rr} 
& $\begin{array}{c}\text { max } \\
\text { (IEC62040) }\end{array}$ & $\begin{array}{r}\text { feedback with } \\
x_{1} \text { measurements }\end{array}$ & $\begin{array}{r}\text { feedback with } \\
x_{1} \text { estimation }\end{array}$ \\
\hline THD & $8 \%$ & $3.81 \%$ & $4.14 \%$ \\
$\mathrm{IHD}_{3}$ & $5 \%$ & $0.002 \%$ & $0.001 \%$ \\
$\mathrm{IHD}_{5}$ & $6 \%$ & $0.003 \%$ & $0.0004 \%$ \\
$\mathrm{IHD}_{7}$ & $5 \%$ & $3.46 \%$ & $3.79 \%$ \\
$\mathrm{IHD}_{9}$ & $1.5 \%$ & $0.63 \%$ & $0.87 \%$ \\
$\mathrm{IHD}_{11}$ & $3.5 \%$ & $1.22 \%$ & $1.23 \%$ \\
$\mathrm{IHD}_{13}$ & $3 \%$ & $0.34 \%$ & $0.27 \%$
\end{tabular}






Figure 6. Transient response to nonlinear load steps compared to overvoltage and undervoltage limits of IEC62040-3 with estimated current feedbcak.
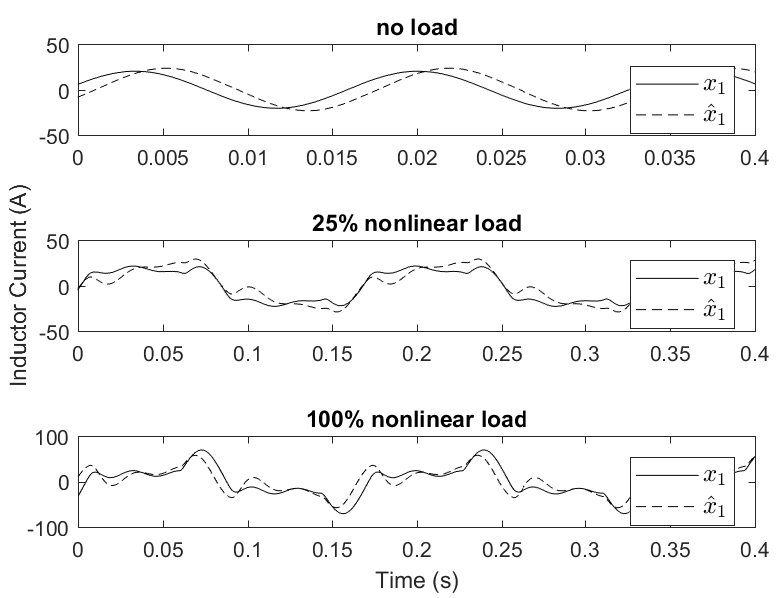

Figure 7. Filter inductor current estimation under different nonlinear loads.

with closed-loop stability assessed a posteriori. Simulation results in PSIM showed compliance to IEC 62040 test standards and a small increase on the THD under current estimation. The experimental validation of the proposed method in an industrial UPS setup is an ongoing work.

\section{REFERENCES}

Boyd, S., El Ghaoui, L., Feron, E., and Balakrishnan, V. (1994). Linear Matrix Inequalities in System and Control Theory, volume 15 of Studies in Applied Mathematics. SIAM, Philadelphia, PA.

Chilali, M., Gahinet, P., and Apkarian, P. (1999). Robust pole placement in LMI regions. IEEE Transactions on Automatic Control, 44(12), 2257-2270.

Commission, I.E. (2011). Uninterruptible power systems (UPS) - Part 3: Method of specifying the performance and test requirements. International Standard IEC62040-3, International Electrotechnical Commission.

Corleta, A., Lorenzini, C., Flores, J.V., and Campestrini, L. (2016). Data-driven control design applied to unin- terruptible power supplies. In 2016 IEEE Conference on Control Applications (CCA), 1312-1317.

Dorf, R.C. and Bishop, R.H. (2000). Modern control systems. Prentice-Hall, Inc., Upper Saddle River, NJ, USA, 9th edition.

Escobar, G., Valdez, A.A., Leyva-Ramos, J., and Mattavelli, P. (2007). Repetitive-based controller for a UPS inverter to compensate unbalance and harmonic distortion. IEEE Transactions on Industrial Electronics, 54(1), 504-510.

Jazwinski, A.H. (1970). Chapter 8 - Applications of Linear Theory. In Stochastic Processes and Filtering Theory, volume 64 of Mathematics in Science and Engineering, 266 - 331. Elsevier.

Lee, Y., Cho, Y., Choe, J., and Choe, G. (2015). Current feedback with decoupling scheme for single-phase UPS using a single current sensor. In 2015 9th International Conference on Power Electronics and ECCE Asia (ICPE-ECCE Asia), 1380-1385.

Middlebrook, R.D. and Cuk, S. (1976). A general unified approach to modelling switching-converter power stages. In 1976 IEEE Power Electronics Specialists Conference, 18-34.

Pereira, L.F.A., Flores, J.V., Bonan, G., Coutinho, D.F., and da Silva, J.M.G. (2014). Multiple resonant controllers for uninterruptible power supplies - a systematic robust control design approach. IEEE Transactions on Industrial Electronics, 61(3), 1528-1538.

Petersen, I.R. and McFarlane, D.C. (1994). Optimal guaranteed cost control and filtering for uncertain linear systems. IEEE Transactions on Automatic Control, 39(9), 1971-1977.

Petersen, I.R., Anderson, B.D.O., and Jonckheere, E.A. (1991). A first principles solution to the non-singular $H_{\infty}$ control problem. International Journal of Robust and Nonlinear Control, 1(3), 171-185.

Prasov, A.A. and Khalil, H.K. (2013). A nonlinear highgain observer for systems with measurement noise in a feedback control framework. IEEE Transactions on Automatic Control, 58(3), 569-580.

Ryan, M.J., Brumsickle, W.E., and Lorenz, R.D. (1997). Control topology options for single-phase UPS inverters. IEEE Transactions on Industry Applications, 33(2), 493-501.

Sangswang, A. and Nwankpa, C.O. (2004). Noise characteristics of DC-DC boost converters: experimental validation and performance evaluation. IEEE Transactions on Industrial Electronics, 51(6), 1297-1304.

Simon, D. (2006). The continuous-time Kalman filter. In Optimal State Estimation, chapter 8, 229-262. WileyBlackwell.

Xie, L. and Soh, Y.C. (1994). Robust Kalman filtering for uncertain systems. Systems \& Control Letters, 22(2), $123-129$.

Xu, S., Wang, J., and Xu, J. (2013). A current decoupling parallel control strategy of single-phase inverter with voltage and current dual closed-loop feedback. IEEE Transactions on Industrial Electronics, 60(4), 13061313.

Ziegler, S., Woodward, R.C., Iu, H.H., and Borle, L.J. (2009). Current sensing techniques: a review. IEEE Sensors Journal, 9(4), 354-376. 